\title{
Diagnosis and Management of Congenital Sensorineural Hearing Loss
}

\section{Michelle M. Chen, MD John S. Oghalai, $M D^{*}$}

\author{
Address \\ "Department of Otolaryngology-Head and Neck Surgery, Stanford University, 801 \\ Welch Road, Palo Alto, CA, 94305-5739, USA \\ Email: joghalai@stanford.edu \\ Published online: 8 July 2016 \\ (C) Springer International Publishing AG 2016
}

This article is part of the Topical Collection on Otolaryngology

Keywords Congenital hearing loss $\cdot$ Sensorineural hearing loss $\cdot$ Deafness $\cdot$ Hearing loss $\cdot$ Cochlear implantation . Diagnosis

\section{Opinion statement}

Hearing loss is the most common sensory disorder in the USA. The diagnosis of congenital hearing loss starts with newborn hearing screening, which is best performed with auditory brainstem evoked responses in order to avoid the risk of missing auditory neuropathy spectrum disorder. A careful history and physical exam can occasionally help reveal the etiology for congenital hearing loss. Imaging studies, either CT temporal bones or MRI of the internal auditory canals without gadolinium, and genetic testing, in particular for connexin 26, connexin 30, and Pendred syndrome, are the most useful diagnostic tests. Management of congenital hearing loss involves early fitting of amplification. Early cochlear implantation, preferably before 2 years of age, should be strongly considered for children with bilateral severe hearing loss.

\section{Introduction}

The incidence of neonatal hearing loss is 1.1 per 1000 infants in the USA and has a prevalence of $3.1 \%$ among children and adolescents [1]. This makes hearing loss the most common sensory disorder and the fourth most common developmental disorder in the USA. Indigent patients with poor access to healthcare are at increased risk of hearing loss [2]. 


\section{Hearing screening}

Prior to the introduction of universal newborn screening, the average age of diagnosis of congenital hearing loss was 2 to 3 years of age [3, 4]. Hearing impairment has a significant impact on communication and spoken language skills. Moreover, there is also a reduction in visual reception and fine motor skill development in hearing-impaired children. The Joint Committee on Infant Hearing now promotes universal screening with state-run systems of early hearing detection and intervention (EHDI) [5]. The goal of the EHDI programs is to screen all infants prior to 1 month of age, those who do not pass the initial screen should have a comprehensive audiologic evaluation by 3 months of age, and interventions should be implemented by 6 months of age [5]. Currently, $97 \%$ of newborns in the USA undergo newborn hearing screening [6•], but only $50.3 \%$ of those who failed the hearing screen had adequate follow-up diagnostic testing [7]. Of those, only $70.2 \%$ had the testing performed prior to 3 months of age [7]. Unilateral hearing loss, later diagnosis, and Medicaid coverage have been show to be factors associated with loss to follow-up [8].

Otoacoustic emissions (OAEs) are often used for newborn hearing screening. The outer hair cells in the cochlea generate forces to amplify incoming sounds and improve auditory thresholds. The outer hair cells also amplify distortions and reflections that naturally occur within the cochlea. These new sounds, created by the cochlea, then back-propagate out of the ear and can be detected by a microphone in the ear canal as an OAE. Thus, OAEs test cochlear function only and do not detect pathology in the auditory nerve or auditory brainstem pathways. This is usually fine because most, but not all, forms of hearing loss are associated with outer hair cell dysfunction and reduced amplification. However, since OAEs depend on sound passing through the ear canal and middle ear and then back out again, any ear canal and middle ear pathology will dramatically affect test results. Otoscopic exams of newborns have demonstrated that the incidence of middle ear effusion or decreased tympanic membrane motility ranges from 0 to $22.7 \%$. In the neonatal intensive care unit (NICU), the presence of middle ear effusions approaches $30 \%$ [9]. In an analysis of neonates with middle ear fluid, only $33 \%$ passed transient-evoked OAE testing. The benefit of OAE testing is that it does not require patient compliance and can be rapidly performed by a technician [10]. A pass on an OAE test indicates that there is hearing sensitivity of $30 \mathrm{~dB}$ HL or better and there is proper functioning of the outer hair cells [11].

Auditory brainstem evoked responses (ABRs) provide another way to assess newborn hearing. In ABR testing, a short tone burst or click is used to stimulate the cochlea, and the electric field potentials generated by the auditory nerve and brainstem are measured. Since a normal ABR result requires a normal middle ear, cochlea, auditory nerve, and auditory brainstem pathways, ABR testing will not only be able to detect hearing loss associated with outer hair cell dysfunction and loss of amplification but also forms of hearing loss due to problems in conveying the sound information to the brain. This is called auditory neuropathy/auditory dyssynchrony or auditory neuropathy spectrum disorder (ANSD), and implies 
that inner hair cells do not effectively detect sounds, that inner hair cells do not adequately convey the detected signal to the afferent auditory nerve, or that the afferent auditory nerve cannot carry the signal to the brain. With auditory neuropathy, a newborn will pass OAE testing, but fail ABR testing. The presence of middle ear effusions does not change the proportion of ears that pass ABR testing because the sound only has to pass through the middle ear one time [12].

The 2007 Joint Committee on Infant Hearing's position statement states that all infants should have a newborn hearing screen prior to 1 month of age [5]. For healthy newborns that do not have prolonged NICU stays, either OAE or ABR is considered satisfactory for screening. Newborns that have been admitted to the NICU for more than 5 days should have ABR testing as part of their hearing screen [5]. Moreover, at least one $\mathrm{ABR}$ is recommended to confirm hearing loss in any children less than 3 years of age with suspected permanent hearing loss. Due to the risk of missing ANSD, the trend is for the routine use of ABR testing for all newborn children. See Fig. 1.

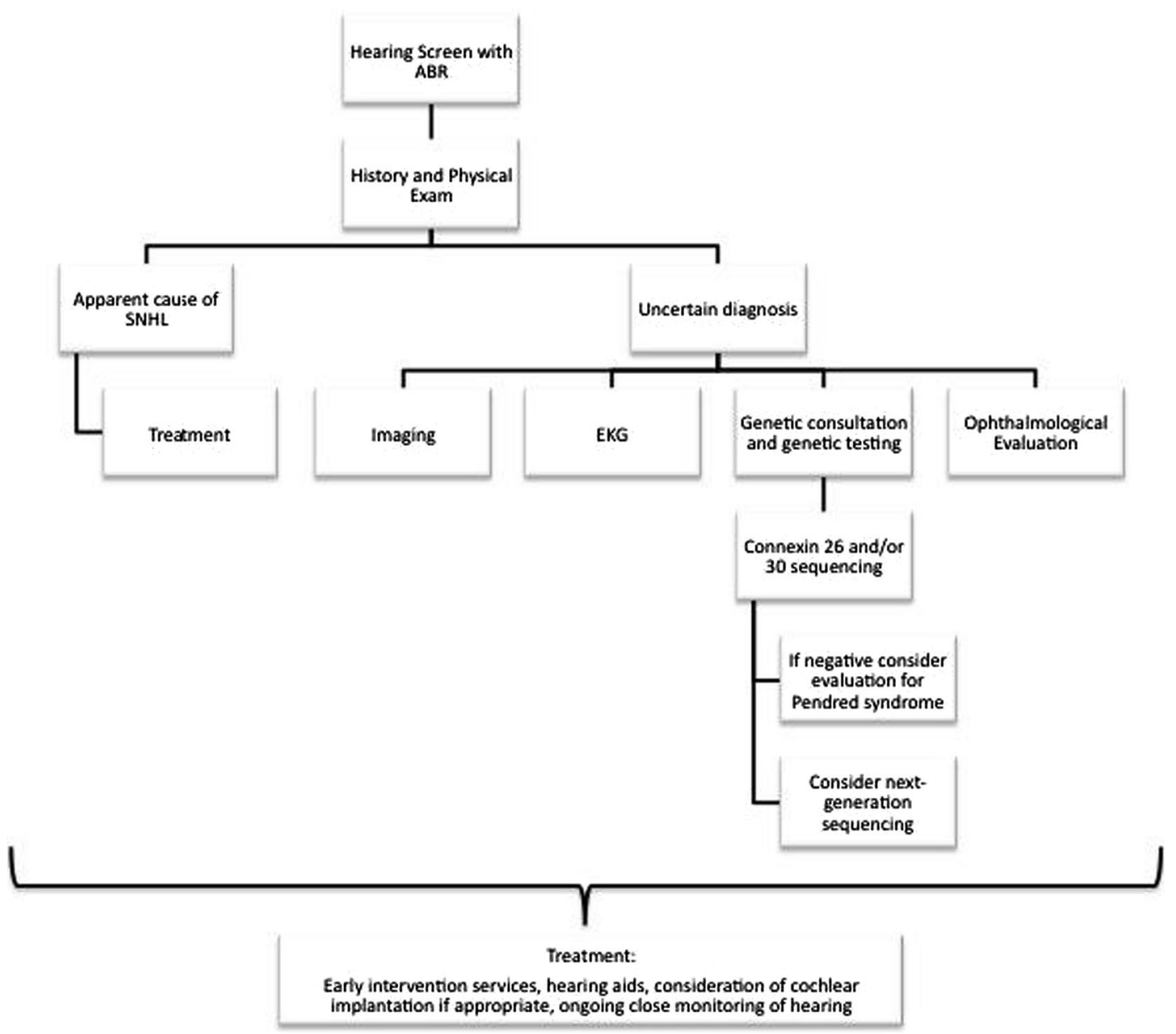

Fig. 1. Diagnostic algorithm for congenital hearing loss. 


\section{Medical evaluation}

\section{History}

Careful history taking can reveal the etiology for congenital hearing loss, especially non-genetic congenital hearing loss. These causes can be grouped into the broad categories of infection, prematurity/NICU stay, and other causes.

Congenital cytomegalovirus (CMV) infection is the most common nongenetic cause of hearing loss. Approximately $10-21 \%$ of all congenital hearing loss is due to congenital CMV infection [13, 14]. Other prenatally acquired maternal infections can also result in congenital hearing loss including rubella, toxoplasmosis, herpes simplex, and syphilis.

The incidence of hearing loss in NICU infants has been shown to range from 0.7 to $4.9 \%$ [15-17]. NICU infants are more likely to have low birth weight, history of birth hypoxia, hyperbilirubinemia, history of extracorporeal membrane oxygenation, history of sepsis, and ototoxic medication use, which are all known risk factors for hearing loss [10, 18]. Hyperbilirubinemia can be associated with neurologic damage in infants and damage to the auditory system primarily involves the brainstem and cranial nerve VIII, which clinically presents as ASND [19]. Farhat et al. compared 2063 NICU infants with 8724 healthy controls in the well-baby nursery and determined that $1.9 \%$ of the NICU infants had confirmed hearing loss compared to $0.3 \%$ of controls [20]. This indicates a sixfold higher rate of hearing loss in NICU infants relative to the healthy controls [20]. Van Dommelen analyzed 10,830 NICU infants in the Netherlands and found that $1.8 \%$ of the infants had hearing loss and the prevalence of hearing loss across NICU centers ranged from 0.7 to $3.7 \%$ [15]. Independent risk factors for hearing loss included craniofacial anomalies, chromosomal/syndromal anomalies, central nervous system and circulatory disorders, congenital infections (toxoplasmosis, rubella, CMV, and herpes simplex), high frequency oxygen ventilation, and $\geq 12$ days of intensive care, and they found that the factors in their model were able to explain about $20 \%$ of the variation in hearing loss across NICU centers [15].

\section{Physical examination}

A careful head and neck physical exam may help reveal anatomic causes of conductive hearing loss such as ear canal atresia, or a middle ear effusion. In addition, malformations that commonly affect the Eustachian tube should strongly raise the suspicion for an effusion such as a cleft lip or palate, Down syndrome, or other craniofacial anomalies [11]. The physical exam may show dysmorphisms that can also help guide the work-up of syndromic sensorineural hearing loss; however, this often requires a multidisciplinary approach because there are over 400 identified syndromes associated with congenital hearing loss and many have complex phenotypes [21].

Diagnostic imaging is one of the most useful diagnostic tests in the work-up of congenital hearing loss. Mafong et al. determined that $39 \%$ of children with sensorineural hearing loss (SNHL) had an abnormality on either temporal bone CT scan or MRI [22]. Preciado et al. demonstrated that the diagnostic yield 
of imaging may vary according to the severity of SNHL; patients with mild SNHL had a lower diagnostic yield (21.1\%) than those with severe to profound hearing loss (29.9\%) [23]. The diagnostic yield of imaging is greater for patients with unilateral hearing loss $(36.7 \%)$ than those with bilateral hearing loss $(24.7 \%)[23]$.

CT scans are useful for evaluating bony anomalies, but do involve exposure to radiation. A systematic review of 50 studies demonstrated that the diagnostic yield for temporal bone CT scans ranges from 7 to $74 \%$ with a pooled diagnostic yield of $30 \%$ and so; on average, four patients need to undergo a CT scan to yield one relevant diagnostic result [24]. Lin et al. found that anomalies in the temporal bone were found in $18 \%$ of children with severe to profound SNHL and the most commonly found anomalies were cochlear dysplasia $(10.2 \%)$, semicircular canal/vestibular dysplasia (10.2\%), IAC/CA anomalies $(7.3 \%)$, and enlarged vestibular aqueducts (5.3\%) [25]. Mathews et al. analyzed 10.9 million people in Australian Medicare records and determined that one in 4000 childhood brain CT scans could have lead to a brain tumor [26]. Thus, given the diagnostic yield of CT scan, a temporal bone CT scan is much more likely to yield a diagnosis than lead to malignancy.

MRI scans provide improved soft tissue resolution. A systematic review of 25 studies of the use of MRI in evaluating pediatric hearing loss reported that the diagnostic yield ranges from 2 to $60 \%$ [27]. The diagnostic yield of MRI for evaluation of auditory spectrum neuropathy was 34 to $100 \%$ [27]. Lin et al. reported that $40 \%$ of children with severe to profound SNHL had abnormal MRI findings and $24 \%$ had MRI findings that could explain their hearing loss [25]. The higher diagnostic yield of MRI relative to CT must be balanced with the increased cost associated with MRI and the need for sedation and additional time compared with CT. 2014 Medi-Cal reimbursement rates for a CT temporal bone were $\$ 212, \$ 953$ for an MRI of the IAC, and $\$ 250$ for sedation during the exam [28].

Laboratory testing

Laboratory tests have limited utility in identifying the etiology of congenital hearing loss. Deklerck et al. analyzed 191 patients with congenital hearing loss and determined that only $45.5 \%$ of patients received laboratory tests and of these, $13.7 \%$ had abnormal results, but only $8.0 \%$ had a relevant result that contributed to the diagnostic work-up [29]. Preciado et al. determined that none of the results of testing ESR, FTA-ABS/VDRL, cholesterol, triglycerides, hemoglobin, platelets, chemistry panel, urinalysis, or thyroid function were of any diagnostic significance for the 474 patients analyzed [23]. Rather than ordering a battery laboratory tests on every patient with congenital hearing loss, they should be used to supplement the diagnostic work-up in select patients.

Like other laboratory testing, routine electrocardiograms (ECGs) on all patients with congenital hearing loss are of low diagnostic yield. However, an ECG that demonstrates a prolonged QT interval such as in Jervell and Lange-Nielsen syndrome may be life-saving [23]. 
Approximately $70 \%$ of genetic hearing loss is nonsyndromic, and of nonsyndromic hearing loss, the majority is autosomal recessive ( $80 \%$ ), followed by autosomal dominant (15\%) and X-linked (1\%) [30]. The most common genetic etiology of congenital SNHL is a mutation in GJB2, which is the gene that encodes connexin 26 [31, 32]. Chan et al. conducted a systematic review that demonstrated that the worldwide prevalence of biallelic GJB2-associated hearing loss was $17.3 \%$ [33]. The rate of biallelic GJB2-associated hearing loss in all patients with congenital hearing loss, nonsyndromic hearing loss, and autosomal recessive nonsyndromic hearing loss was $16.9,18.1$, and $21.3 \%$, respectively [33]. Among patients with biallelic GJB2-associated hearing loss, the $35 \mathrm{delG}$ mutation accounts for $57 \%$ of all alleles [33]. If nonsyndromic hearing loss is suspected, one should consider single-gene testing for GLB2 [30].

Genetic work-up of syndromic hearing loss should be based on the suspected syndrome. The most common causes of syndromic hearing loss are Usher syndrome, which is associated with visual loss, and Pendred syndrome, which has enlarged vestibular aquaducts detectable by imaging and is often associated with hypothyroidism that manifests in the teenage years.

There have been rapid advances in high-throughput sequencing that now allow sequencing of multiple genes that cause SNHL. Often providers may use a two-tiered approach where testing starts with a first tier test that includes the most common genes associated with SNHL, including GJB2 (connexin 26) and GJB6 (connexin 30). If first tier testing is negative, then providers can proceed to second tier testing with next-generation sequencing in order to screen for multiple genes associated with hearing loss.

Ophthalmologic evaluation found abnormalities in 35.7 to $39 \%$ of children $[25,29]$. Forty-three point nine percent of the abnormalities revealed on ophthalmologic work-up were relevant for the diagnostic work-up [29]. Furthermore, children with hearing loss are dependent more on their visual capacity, so ophthalmologic evaluation should be done in all children with congenital hearing loss.

\section{Treatment}

Age at fitting of amplification is the strongest influence on speech perception, speech production, and spoken language [34]. Factors that influenced hearing aid use by children included maternal education level, chronologic age, and degree of hearing loss $[35,36]$. There is a 2 -h difference in hearing aid use between mothers with a college education and those with a high school education or less, emphasizing the need for focused counseling on hearing aid adherence, especially for families with lower education levels [35]. Children with milder losses are less likely to wear their hearing aids in public situations than children with more severe hearing loss [35]. However, even mild hearing loss is significantly associated with poorer academic performance [37].

The preferred choice of hearing aid for pediatric patients is the behind the ear (BTE) style. This style is recommended by the American Academy of Audiology 
for young children because there is less of a swallowing risk compared with inthe-ear (ITE) hearing aids [38]. For all children with bilateral hearing loss, the recommendation is for bilateral hearing aids [38]. The advantage of bilateral hearing aids is the enhancement of binaural hearing, improved auditory localization, and speech understanding in noise.

During the first 2 years of hearing aid use, the Pediatric Working Group suggests that audiologic appointments should be made every 3 months to monitor auditory status and hearing aid fitting due to rapid ear canal growth during early childhood [6•]. This timeline can be adjusted if there are any new concerns or there is increased risk for progressive hearing loss.

The cochlear implant directly stimulates the spiral ganglion cells, which are the first order neurons of the auditory pathway. According to the Food and Drug Administration (FDA), approximately 324,200 people worldwide have received implants as of December 2012 [39]. Cochlear implantation has a low rate of complications, and major complications are rare, including facial nerve injury $(0.39 \%)$, perilymphatic gusher/cerebrospinal fluid fistula $(0.25 \%)$, and meningitis $(0.11 \%)$ [ 40$]$. Cochlear implants have been FDA-approved for use in children beginning at 12 months of age. However, the literature suggests that there continues to be a trend toward improved auditory rehabilitation and language development with earlier implantation. Colletti et al. analyzed the long-term outcomes of pediatric patients with cochlear implants and found that patients implanted before 12 months have greater receptive language growth than those implanted at or after 12 months of age [41]. Nine years after implantation, $100 \%$ of the children implanted before 12 months, $28 \%$ of those implanted at $12-23$ months, and $20 \%$ of those implanted at 2436 months scored in the $76-100^{\text {th }}$ percentile on speech reception [41].

The best imaging for pediatric cochlear implant candidates is unclear. The majority of anatomic anomalies that would affect cochlear implantation can be seen on either CT or MRI. However, MRI is superior for detecting cochlear nerve dysplasia and other intracranial pathologies that may impact the implantation decision-making process.

Cochlear implantation has been demonstrated to be clinically effective in children with severe to profound bilateral hearing loss as well as cost-effective [42]. Niparko et al. conducted a prospective longitudinal multicenter evaluation of spoken language outcomes after implantation in children with severe to profound SNHL and found that the use of cochlear implants in these children was associated with improved spoken language development than would be estimated from their pre-implantation scores on measures of spoken language comprehension and expression [43].

The benefit of cochlear implantation in children with unilateral sensorineural hearing loss is uncertain. Reeder et al. reported that children with unilateral hearing loss have significant differences from their normal-hearing peers in terms of speech perception and localization [44]. Children with unilateral hearing loss have been shown to have worse language skills than their siblings with normal hearing [45]. Peters et al. conducted a systematic review of 296 articles on cochlear implantation in children with unilateral hearing loss and found mixed results with regards to sound localization and only one study 
demonstrated improvement in speech and language development [46•]. Most patients had improvements in speech perception in noise, but results were not all statistically significant due to the small sample sizes [46•]. The role of implantation in single-sided deafness is still an active area of research, both in children and in adults.

\section{Auditory brainstem implants}

Auditory brainstem implants bypass the cochlear nerve and stimulate the second-order auditory neurons in the cochlear nucleus [47]. The first successful auditory brainstem implant was placed in 1979, and the first FDA approval for an auditory brainstem implant was received in 2000 for a Cochlear Corporation implant to be used in patients with neurofibromatosis type 2 (NF2). Non-NF2 patients who have received the brainstem implants in Europe include those with severe cochlear or cochlear nerve malformations or aplasia, severe cochlear ossification, and temporal bone fractures associated with traumatic cochlear nerve avulsion [48]. Patients with NF2 have open-set sound only sentence recognition scores of 5 to $31 \%$, while non-NF2 patients have been shown to have open-set speech perception scores of 10 to $100 \%$ [4850]. However, in general, the results of auditory brainstem implants to date have been unreliable.

\section{Conclusion}

The key to the management of congenital hearing loss is early diagnosis and early intervention. Evaluation of congenital hearing loss starts with newborn hearing screenings with ABRs and includes a thorough history and physical exam, diagnostic imaging, electrocardiography, genetic testing and consultation, and ophthalmological exam. Treatment primarily consists of amplification and cochlear implantation, combined with speech and language therapy in an appropriate educational environment.

\section{Acknowledgments}

This works was in part supported by the NIH R01 DC010075.

\section{Compliance with Ethical Standards}

\section{Conflict of Interest}

John S. Oghalai reports grants from the NIH (R01 DC010075), during the conduct of the study.

Michelle M. Chen declares that she has no conflict of interest.

Human and Animal Rights and Informed Consent

This article does not contain any studies with human or animal subjects performed by any of the authors. 


\section{References and Recommended Reading}

Papers of particular interest, published recently have been highlighted as

- $\quad$ Of importance

1. Mehra S, Eavey RD, Keamy DG. The epidemiology of hearing impairment in the United States: newborns, children, and adolescents. Otolaryngol-Head Neck Surg. 2009;140(4):461-72.

2. Oghalai JS, Chen L, Brennan ML, Tonini R, Manolidis $\mathrm{S}$. Neonatal hearing loss in the indigent. Laryngoscope. 2002;112(2):281-6.

3. Coplan J. Deafness: ever heard of it? delayed recognition of permanent hearing loss. Pediatrics. 1987;79(2):206-13.

4. Naarden KV, Decouflé P, Caldwell K. Prevalence and characteristics of children with serious hearing impairment in Metropolitan Atlanta, 1991-1993. Pediatrics. 1999;103(3):570-5.

5. Joint Committee on Infant Hearing. Year 2007 position statement: principles and guidelines for early hearing detection and intervention programs. Pediatrics. 2007;120(4):898-921.

6. Tharpe AM, Gustafson S. Management of children with mild, moderate, and moderately severe sensorineural hearing loss. Otolaryngol Clin North Am. 2015;48(6):983-94.

Detailed review of the management of children with different levels of sensorineural hearing loss.

7. Early hearing detection and intervention among infants-hearing screening and follow-up survey, United States, 2005-2006 and 2009-2010 [Internet]. [cited 2016 Mar 15]. Available from: http://www.cdc. gov.laneproxy.stanford.edu/mmwr/preview/ mmwrhtml/su6302a4.htm.

8. Spivak L, Sokol H, Auerbach C, Gershkovich S. Newborn hearing screening follow-up: factors affecting hearing aid fitting by 6 months of age. Am J Audiol. 2009;18(1):24-33.

9. Balkany TJ, Berman SA, Simmons MA, Jafek BW. Middle ear effusions in neonates. Laryngoscope. 1978;88(3):398-405.

10. Cristobal R, Oghalai JS. Hearing loss in children with very low birth weight: current review of epidemiology and pathophysiology. Arch Dis Child - Fetal Neonatal Ed. 2008;93(6):F462-8.

11. Jerry J, Oghalai JS. Towards an etiologic diagnosis: assessing the patient with hearing loss [Internet]. vol. 70. Karger Publishers; 2011 [cited 2016 Mar 15]. Available from: http://www.karger.com. laneproxy.stanford.edu/Article/Abstract/322468.

12. Doyle KJ, Rodgers P, Fujikawa S, Newman E. External and middle ear effects on infant hearing screening test results. Otolaryngol - Head Neck Surg.

2000;122(4):477-81.
13. Morton CC, Nance WE. Newborn hearing screening - a silent revolution. N Engl J Med. 2006;354(20):2151-64.

14. Fowler KB. Congenital cytomegalovirus infection: audiologic outcome. Clin Infect Dis. 2013;57 suppl 4:S182-4.

15. Van Dommelen P, Mohangoo A, Verkerk P, Van Der Ploeg C, Van Straaten H, The Dutch NICU Neonatal Hearing Screening Working Group. Risk indicators for hearing loss in infants treated in different neonatal intensive care units. Acta Paediatr. 2010;99(3):344-9.

16. Coenraad S, Goedegebure A, van Goudoever JB, Hoeve LJ. Risk factors for sensorineural hearing loss in NICU infants compared to normal hearing NICU controls. Int J Pediatr Otorhinolaryngol. 2010;74(9):999-1002.

17. Colella-Santos MF, Hein T, Dinizs A, de Souza GL, Do Amaral MIR, Casali RL. Newborn hearing screening and early diagnostic in the NICU, newborn hearing screening and early diagnostic in the NICU. BioMed Res Int BioMed Res Int. 2014;2014:e845308.

18. Bamiou D e, Macardle B, Bitner-Glinzicz M, Sirimanna T. Aetiological investigations of hearing loss in childhood: a review. Clin Otolaryngol Allied Sci. 2000;25(2):98-106.

19. Olds C, Oghalai JS. Audiologic impairment associated with bilirubin-induced neurologic damage. Semin Fetal Neonatal Med. 2015;20(1):42-6.

20. Farhat A, Ghasemi MM, Akhondian J, Mohammadzadeh A, Esmaeili H, Amiri R, et al. Comparative study of hearing impairment among healthy and intensive care unit neonates in Mashhad, North East Iran. Iran J Otorhinolaryngol. 2015;27(81):273-7.

21. Deltenre P, Van Maldergem L. Chapter 158-Hearing loss and deafness in the pediatric population: causes, diagnosis, and rehabilitation. In: Olivier Dulac ML and HBS, editor. Handbook of clinical neurology [Internet]. Elsevier; 2013 [cited 2016 Mar 15]. p. 152738. (Pediatric neurology Part III; vol. 113). Available from: http://www.sciencedirect.com/science/article/ pii/B978044459565200023X.

22. Mafong DD, Shin EJ, Lalwani AK. Use of laboratory evaluation and radiologic imaging in the diagnostic evaluation of children with sensorineural hearing loss. Laryngoscope. 2002;112(1):1-7.

23. Preciado DA, Lim LHY, Cohen AP, Madden C, Myer D, Ngo C, et al. A diagnostic paradigm for childhood idiopathic sensorineural hearing loss.

Otolaryngol-Head Neck Surg. 2004;131(6):804-9. 
24. Chen JX, Kachniarz B, Shin JJ. Diagnostic yield of computed tomography scan for pediatric hearing loss: a systematic review. Otolaryngol-Head Neck Surg. 2014;151(5):718-39.

25. Lin JW, Chowdhury N, Mody A, Tonini R, Emery C, Haymond J, et al. Comprehensive diagnostic battery for evaluating sensorineural hearing loss in children. Otol Neurotol. 2011;32(2):259-64.

26. Mathews JD, Forsythe AV, Brady Z, Butler MW, Goergen SK, Byrnes GB. Cancer risk in 680000 people exposed to computed tomography scans in childhood or adolescence: data linkage study of 11 million Australians. BMJ. 2013;346(may21 1):f2360.

27. Kachniarz B, Chen JX, Gilani S, Shin JJ. Diagnostic yield of MRI for pediatric hearing loss: a systematic review. Otolaryngol-Head Neck Surg. 2015;152(1):5-22.

28. Tamplen M, Schwalje A, Lustig L, Alemi AS, Miller ME. Utility of preoperative computed tomography and magnetic resonance imaging in adult and pediatric cochlear implant candidates. Laryngoscope. 2015;n/a-n/a

29. Deklerck AN, Acke FR, Janssens S, De Leenheer EMR. Etiological approach in patients with unidentified hearing loss. Int J Pediatr Otorhinolaryngol. 2015;79(2):216-22.

30. Loss AWG on U of GEG for the ED of $\mathrm{CH}$, Committee for the PP and G. American College of Medical Genetics and Genomics guideline for the clinical evaluation and etiologic diagnosis of hearing loss. Genet Med. 2014;16(4):347-55.

31. Kelsell DP, Dunlop J, Stevens HP, Lench NJ, Liang JN, Parry G, et al. Connexin 26 mutations in hereditary non-syndromic sensorineural deafness. Nature. 1997;387(6628):80-3.

32. Tang H-Y, Fang P, Ward PA, Schmitt E, Darilek S, Manolidis S, et al. DNA sequence analysis of GJB2, encoding connexin 26: observations from a population of hearing impaired cases and variable carrier rates, complex genotypes, and ethnic stratification of alleles among controls. Am J Med Genet A. 2006;140A(22):2401-15.

33. Chan DK, Chang KW. GJB2-associated hearing loss: systematic review of worldwide prevalence, genotype, and auditory phenotype. Laryngoscope.

2014;124(2):E34-53.

34. Sininger YS, Grimes A, Christensen E. Auditory development in early amplified children: factors influencing auditory-based communication outcomes in children with hearing loss. Ear Hear. 2010;31(2):166-85.

35. Walker EA, Spratford M, Moeller MP, Oleson J, Ou H, Roush $\mathrm{P}$, et al. Predictors of hearing aid use time in children with mild-severe hearing loss. Lang Speech Hear Serv Sch. 2013;44(1):73.

36. Holte L, Walker E, Oleson J, Spratford M, Moeller MP, Roush $\mathrm{P}$, et al. Factors influencing follow-up to newborn hearing screening for infants who are hard of hearing. Am J Audiol. 2012;21(2):163-74.
37. Khairi Md Daud M, Noor RM, Rahman NA, Sidek DS, Mohamad A. The effect of mild hearing loss on academic performance in primary school children. Int J Pediatr Otorhinolaryngol. 2010;74(1):67-70.

38. Pediatric Amplication Guidelines [Internet]. [cited 2016 Mar 14]. Available from: http://audiology-web. s3.amazonaws.com/migrated/ PediatricAmplificationGuidelines.pdf_ 539975b3e7e9f1.74471798.pdf.

39. Bobsin LL, Houston KT. Communication assessment and intervention: implications for pediatric hearing loss. Otolaryngol Clin North Am. 2015;48(6):1081-95.

40. Vincenti V, Bacciu A, Guida M, Marra F, Bertoldi B, Bacciu $S$, et al. Pediatric cochlear implantation: an update. Ital J Pediatr. 2014;40:72.

41. Colletti L. Long-term follow-up of infants (411 months) fitted with cochlear implants. Acta Otolaryngol (Stockh). 2009;129(4):361-6.

42. Bond M, Mealing S, Anderson R, Elston J, Weiner G, Taylor RS, et al. The effectiveness and cost-effectiveness of cochlear implants for severe to profound deafness in children and adults: a systematic review and economic model. Health Technol Assess Winch Engl. 2009;13(44):1-330.

43. Niparko JK, Tobey EA, Thal DJ, et al. SPoken language development in children following cochlear implantation. JAMA. 2010;303(15):1498-506.

44. Reeder RM, Cadieux J, Firszt JB. Quantification of speech-in-noise and sound localisation abilities in children with unilateral hearing loss and comparison to normal hearing peers. Audiol Neurotol. 2015;20(1):31-7.

45. Lieu JEC, Tye-Murray N, Karzon RK, Piccirillo JF. Unilateral hearing loss is associated with worse speechlanguage scores in children. Pediatrics. 2010;125(6):e1348-55.

46.• Peters JPM, Ramakers GGJ, Smit AL, Grolman W. Cochlear implantation in children with unilateral hearing loss: a systematic review. Laryngoscope. 2016;126(3):713-21.

Review of the controversies in the literature surrounding cochlear implantation in children with unilateral hearing loss.

47. Puram SV, Lee DJ. Pediatric auditory brainstem implant surgery. Otolaryngol Clin North Am. 2015;48(6):1117-48.

48. Colletti V, Shannon R, Carner M, Veronese S, Colletti L. Outcomes in nontumor adults fitted with the auditory brainstem implant: 10 years' experience. Otol Neurotol. 2009;30(5):614-8.

49. Colletti V. Auditory outcomes in tumor vs. nontumor patients fitted with auditory brainstem implants. Adv Otorhinolaryngol. 2006;64:167-85.

50. Colletti L, Shannon RV, Colletti V. The development of auditory perception in children after auditory brainstem implantation. Audiol Neurootol. 2014;19(6):386-94. 